PROCEEDINGS OF THE

AMERICAN MATHEMATICAL SOCIETY

Volume 141, Number 5, May 2013, Pages 1679-1692

S 0002-9939(2012)11443-1

Article electronically published on November 2, 2012

\title{
A MAXIMAL FUNCTION CHARACTERIZATION OF THE HARDY SPACE FOR THE GAUSS MEASURE
}

\author{
GIANCARLO MAUCERI, STEFANO MEDA, AND PETER SJÖGREN
}

(Communicated by Michael T. Lacey)

\begin{abstract}
An atomic Hardy space $H^{1}(\gamma)$ associated to the Gauss measure $\gamma$ in $\mathbb{R}^{n}$ has been introduced by the first two authors. We first prove that it is equivalent to use $(1, r)$ - or $(1, \infty)$-atoms to define this $H^{1}(\gamma)$. For $n=1$, a maximal function characterization of $H^{1}(\gamma)$ is found. In arbitrary dimension, we give a description of the nonnegative functions in $H^{1}(\gamma)$ and use it to prove that $L^{p}(\gamma) \subset H^{1}(\gamma)$ for $1<p \leq \infty$.
\end{abstract}

\section{INTRODUCTION}

Denote by $\gamma$ the Gauss measure on $\mathbb{R}^{n}$, i.e., the probability measure with density $\gamma_{0}(x)=\pi^{-n / 2} \mathrm{e}^{-|x|^{2}}$ with respect to the Lebesgue measure $\lambda$. Harmonic analysis on the measured metric space $\left(\mathbb{R}^{n}, d, \gamma\right)$, where $d$ denotes the Euclidean distance on $\mathbb{R}^{n}$, has been the object of many investigations. In particular, efforts have been made to study operators related to the Ornstein-Uhlenbeck semigroup, with emphasis on maximal operators [33, 16, 27, 13, 21, 22, Riesz transforms [29, 14, 28, 32, 30, 17, 15, 8, 9, 10, 31, 35, 7, 24, and functional calculus [11, 12, 18, 25.

In 24 the first two authors defined an atomic Hardy-type space $H^{1}(\gamma)$ and a space $B M O(\gamma)$ of functions of bounded mean oscillation, associated to $\gamma$. We briefly recall their definitions. A closed Euclidean ball $B$ is called admissible at scale $s>0$ if

$$
r_{B} \leq s \min \left(1,1 /\left|c_{B}\right|\right)
$$

here and in the sequel $r_{B}$ and $c_{B}$ denote the radius and the centre of $B$, respectively. We denote by $\mathcal{B}_{s}$ the family of all balls admissible at scale $s$. For the sake of brevity, we shall refer to balls in $\mathcal{B}_{1}$ simply as admissible balls. Further, $B$ will be called maximal admissible if $r_{B}=\min \left(1,1 /\left|c_{B}\right|\right)$.

Now let $r \in(1, \infty]$. A Gaussian $(1, r)$-atom is either the constant function 1 or a function $a$ in $L^{r}(\gamma)$ supported in an admissible ball $B$ and such that

$$
\int a \mathrm{~d} \gamma=0 \quad \text { and } \quad\|a\|_{r} \leq \gamma(B)^{1 / r-1} ;
$$

here and in the whole paper, $\|\cdot\|_{r}$ denotes the norm in $L^{r}(\gamma)$. In the latter case, we say that the atom $a$ is associated to the ball $B$. The space $H^{1, r}(\gamma)$ is then the vector

Received by the editors February 10, 2011 and, in revised form, September 7, 2011.

2010 Mathematics Subject Classification. Primary 42B30, 42B35; Secondary 42C10.

Key words and phrases. Gaussian measure, Gaussian Hardy space, maximal function, atomic decomposition.

This work was partially supported by PRIN 2009 "Analisi Armonica".

(C) 2012 American Mathematical Society Reverts to public domain 28 years from publication 
space of all functions $f$ in $L^{1}(\gamma)$ that admit a decomposition of the form $\sum_{j} \lambda_{j} a_{j}$, where the $a_{j}$ are Gaussian $(1, r)$-atoms and the sequence of complex numbers $\left\{\lambda_{j}\right\}$ is summable. The norm of $f$ in $H^{1, r}(\gamma)$ is defined as the infimum of $\sum_{j}\left|\lambda_{j}\right|$ over all representations of $f$ as above.

In 24 the spaces $H^{1, r}(\gamma)$ were defined and proved to coincide for all $1<r<\infty$, with equivalent norms. In Section 2 we complement this by proving that they coincide also with the space $H^{1, \infty}(\gamma)$. Once this is established, we shall denote the space by $H^{1}(\gamma)$ and use the $H^{1, \infty}(\gamma)$ norm. Further, we shall frequently write atom for the $(1, \infty)$-atom.

The space $B M O(\gamma)$ consists of all functions $f$ in $L^{1}(\gamma)$ such that

$$
\sup _{B \in \mathcal{B}_{1}} \frac{1}{\gamma(B)} \int_{B}\left|f-f_{B}\right| \mathrm{d} \gamma<\infty
$$

where $f_{B}$ denotes the mean value of $f$ on $B$, taken with respect to the Gauss measure. The norm of a function in $B M O(\gamma)$ is

$$
\|f\|_{B M O(\gamma)}=\|f\|_{1}+\sup _{B \in \mathcal{B}_{1}} \frac{1}{\gamma(B)} \int_{B}\left|f-f_{B}\right| \mathrm{d} \gamma .
$$

If, in the definitions of $H^{1}(\gamma)$ and $B M O(\gamma)$, we replace the family $\mathcal{B}_{1}$ of admissible balls at scale 1 by $\mathcal{B}_{s}$ for any fixed $s>0$, we obtain the same spaces with equivalent norms; see [24]. We remark that a similar $H^{1}-B M O$ theory for more general measured metric spaces has been developed by A. Carbonaro and the first two authors in [1, 2, 3. See also the papers [19, 20] of L. Liu and D. Yang for related results.

The main motivation for introducing these two spaces was to provide endpoint estimates for singular integrals associated to the Ornstein-Uhlenbeck operator $\mathcal{L}=$ $-(1 / 2) \Delta+x \cdot \nabla$, a natural self-adjoint Laplacian on $L^{2}(\gamma)$. Indeed, in 24 the first two authors proved that the imaginary powers of $\mathcal{L}$ are bounded from $H^{1}(\gamma)$ to $L^{1}(\gamma)$ and from $L^{\infty}(\gamma)$ to $B M O(\gamma)$ and that Riesz transforms of the form $\nabla^{\alpha} \mathcal{L}^{-|\alpha|}$ and of any order are bounded from $L^{\infty}(\gamma)$ to $B M O(\gamma)$. In a recent paper [26], the authors proved that boundedness from $H^{1}(\gamma)$ to $L^{1}(\gamma)$ and from $L^{\infty}(\gamma)$ to $B M O(\gamma)$ holds for any first-order Riesz transform in dimension one, but not always in higher dimensions.

The definition of the space $H^{1}(\gamma)$ closely resembles the atomic definition of the classical Hardy space $H^{1}(\lambda)$ on $\mathbb{R}^{n}$ endowed with the Lebesgue measure $\lambda$, but there are two basic differences. First, the measured metric space $\left(\mathbb{R}^{n}, d, \gamma\right)$ is nondoubling. Further, except for the constant atom, a Gaussian atom must have "small support", i.e., support contained in an admissible ball. Despite these differences, $H^{1}(\gamma)$ shares many of the properties of $H^{1}(\lambda)$. In particular, the topological dual of $H^{1}(\gamma)$ is isomorphic to $B M O(\gamma)$, an inequality of John-Nirenberg type holds for functions in $B M O(\gamma)$ and the spaces $L^{p}(\gamma)$ are intermediate spaces between $H^{1}(\gamma)$ and $B M O(\gamma)$ for the real and the complex interpolation methods.

It is well known that the classical Hardy space $H^{1}(\lambda)$ can be defined in at least three different ways: the atomic definition, the maximal definition and the definition based on Riesz transforms 6, 34.

As shown in [26], in higher dimensions the first-order Ornstein-Uhlenbeck Riesz transforms $\partial_{j} \mathcal{L}^{-1 / 2}$ are unbounded from $H^{1}(\gamma)$ to $L^{1}(\gamma)$; here $\partial_{j}=\partial / \partial_{x_{j}}$. Thus $H^{1}(\gamma)$ does not coincide with the space of all functions in $L^{1}(\gamma)$ such that $\partial_{j} \mathcal{L}^{-1 / 2} f$ $\in L^{1}(\gamma)$ for $j=1, \ldots, n$. 
This paper arose from the desire to find a maximal characterization of the space $H^{1}(\gamma)$. We recall that the classical space $H^{1}(\lambda)$ can be characterized as the space of all functions $f$ in $L^{1}(\lambda)$ whose grand maximal function

$$
\mathcal{M} f(x)=\sup \left\{\left|\phi_{t} * f(x)\right|: \phi \in \Phi, t>0\right\}
$$

is also in $L^{1}(\lambda)$. Here $\Phi=\left\{\phi \in C_{c}^{1}(B(0,1)):\left|D^{\alpha} \phi\right| \leq 1\right.$ for $\left.|\alpha|=0,1\right\}$ and $\phi_{t}(x)=$ $t^{-n} \phi(x / t)$.

To characterize $H^{1}(\gamma)$, we introduce the local grand maximal function defined on $L_{\text {loc }}^{1}\left(\mathbb{R}^{n}, \gamma\right)$ by

$$
\mathcal{M}_{\text {loc }} f(x)=\sup \left\{\left|\phi_{t} * f(x)\right|: \phi \in \Phi, 0<t<\min (1,1 /|x|)\right\} .
$$

In Section 3 we shall prove that, in arbitrary dimensions, $f \in H^{1}(\gamma)$ implies $\mathcal{M}_{\text {loc }} f \in L^{1}(\gamma)$. Moreover, in dimension one, $H^{1}(\gamma)$ can be characterized as the space of all functions $f$ in $L^{1}(\gamma)$ satisfying $\mathcal{M}_{\text {loc }} f \in L^{1}(\gamma)$ and the following additional global condition:

$$
E(f)=\int_{0}^{\infty} x\left(\left|\int_{x}^{\infty} f \mathrm{~d} \gamma\right|+\left|\int_{-\infty}^{-x} f \mathrm{~d} \gamma\right|\right) \mathrm{d} \lambda(x)<\infty .
$$

This is Theorem 3.3 below.

Roughly speaking, if we interpret a function $f$ as a density of electrical charge on the real line, this global condition says that the positive and negative charges nearly balance out, so that the net charges inside the intervals $(-\infty,-x)$ and $(x, \infty)$ decay sufficiently fast as $x$ approaches $+\infty$. The condition is violated when the distance between the positive and the negative charges increases too much or the charges do not decay sufficiently fast at infinity. For instance, let $\left(a_{n}\right)_{1}^{\infty}$ and $\left(a_{n}^{\prime}\right)_{1}^{\infty}$ be increasing sequences in $(2, \infty)$ such that

$$
a_{n}+2 / a_{n}<a_{n}^{\prime} \text { and } a_{n}^{\prime}+2 / a_{n}^{\prime}<a_{n+1}<2 a_{n}
$$

for all $n$. Then set

$$
f=\sum_{1}^{\infty} c_{n}\left(\frac{\chi_{\left(a_{n}, a_{n}+1 / a_{n}\right)}}{\gamma\left(a_{n}, a_{n}+1 / a_{n}\right)}-\frac{\chi_{\left(a_{n}^{\prime}, a_{n}^{\prime}+1 / a_{n}^{\prime}\right)}}{\gamma\left(a_{n}^{\prime}, a_{n}^{\prime}+1 / a_{n}^{\prime}\right)}\right)
$$

for some $c_{n}>0$. One easily verifies that $\mathcal{M}_{\text {loc }} f \in L^{1}(\gamma)$ if and only if $\sum c_{n}<\infty$. But the global condition $E(f)<\infty$ is equivalent to $\sum c_{n} a_{n}\left(a_{n}^{\prime}-a_{n}\right)<\infty$, which is here a stronger condition.

We have not been able to find a similar characterization of $H^{1}(\gamma)$ in higher dimensions. However, in Section 4 we prove in all dimensions that if $\mathcal{M}_{\text {loc }} f \in L^{1}(\gamma)$ and the function $f$ satisfies the stronger global condition

$$
E_{+}(f)=\int|x|^{2}|f(x)| \mathrm{d} \gamma(x)<\infty,
$$

then $f \in H^{1}(\gamma)$. Observe that for $n=1$ and $f \geq 0$, Fubini's theorem implies that the conditions $E(f)<\infty$ and $E_{+}(f)<\infty$ are equivalent. In arbitrary dimensions, $E_{+}(f)$ can be used to characterize the nonnegative functions in $H^{1}(\gamma)$; see Theorem 4.2. This also leads to a simple proof of the inclusions $L^{p}(\gamma) \subset H^{1}(\gamma)$ and $B M O(\gamma) \subset L^{p^{\prime}}(\gamma)$ for $1<p \leq \infty$.

We end the introduction with some technical observations and notation. In the following we use repeatedly the fact that on admissible balls at a fixed scale $s$, 
the Gauss and the Lebesgue measures are equivalent; i.e., there exists a positive constant $C(s)$ such that for every measurable subset $E$ of $B \in \mathcal{B}_{s}$,

$$
C(s)^{-1} \gamma(E) \leq \gamma_{0}\left(c_{B}\right) \lambda(E) \leq C(s) \gamma(E) .
$$

In particular this implies that the Gauss measure is doubling on balls in $\mathcal{B}_{s}$, with a constant that depends on $s$ (see [24, Prop. 2.1]). Further, it is straightforward to see that if $B^{\prime} \subset B$ are two balls and $B \in \mathcal{B}_{s}$, then $B^{\prime}$ is also in $\mathcal{B}_{s}$.

Given a ball $B$ in $\mathbb{R}^{n}$ and a positive number $\rho$, we shall denote by $\rho B$ the ball with the same centre and with radius $\rho r_{B}$.

In the following $C$ denotes a positive constant whose value may change from occurrence to occurrence and which depends only on the dimension $n$, except when otherwise explicitly stated.

\section{Coincidence of $H^{1, \infty}(\gamma)$ And $H^{1, r}(\gamma)$}

First we need a lemma which will play a role also in the maximal characterization. It deals with the classical Hardy space $H^{1}(\lambda)$ with respect to the Lebesgue measure and the associated standard $(1, \infty)$-atoms, called Lebesgue atoms below.

Lemma 2.1. If $g \in H^{1}(\lambda)$ and the support of $g$ is contained in a ball $B$, then $g$ has an atomic decomposition $g=\sum_{k} \lambda_{k} a_{k}$, where the $a_{k}$ are Lebesgue $(1, \infty)$-atoms associated to balls contained in $2 B$ and

$$
\sum_{k}\left|\lambda_{k}\right| \leq C\|g\|_{H^{1}(\lambda)} .
$$

The lemma can be proved by applying [23, Theor. 4.13] to the space of homogeneous type $B$, endowed with Euclidean distance and Lebesgue measure. Some related results can be found in [4] and [5].

Theorem 2.2. For every $r$ in $(1, \infty)$, the spaces $H^{1, r}(\gamma)$ and $H^{1, \infty}(\gamma)$ coincide, with equivalent norms.

Proof. In this proof, the constants $C$ may depend on $r$ and $n$. Since any Gaussian $(1, \infty)$-atom is also a Gaussian $(1, r)$-atom, $H^{1, \infty}(\gamma)$ is a subspace of $H^{1, r}(\gamma)$ and $\|f\|_{H^{1, r}(\gamma)} \leq\|f\|_{H^{1, \infty}(\gamma)}$. Conversely, suppose that $a$ is a Gaussian $(1, r)$-atom associated to the ball $B \in \mathcal{B}_{1}$. Then the function $a \gamma_{0}$ is a multiple of a Lebesgue (1,r)-atom. Indeed, $\int a \gamma_{0} \mathrm{~d} \lambda=\int a \mathrm{~d} \gamma=0$ and, by the equivalence of the Gauss and Lebesgue measures on admissible balls,

$$
\left\|a \gamma_{0}\right\|_{L^{r}(\lambda)} \leq C \lambda(B)^{1 / r-1} .
$$

Hence, $a \gamma_{0}$ is in $H^{1}(\lambda)$ with norm at most $C$. By Lemma 2.1, it has a decomposition

$$
a \gamma_{0}=\sum_{j} \lambda_{j} \alpha_{j}
$$

where each $\alpha_{j}$ is a Lebesgue $(1, \infty)$-atom associated to a ball $B_{j}$ contained in $2 B$. Moreover

$$
\sum_{j}\left|\lambda_{j}\right| \leq C
$$

and each $B_{j}$ is admissible at scale 2. Define $a_{j}=\alpha_{j} \gamma_{0}^{-1}$. Then $\int a_{j} \mathrm{~d} \gamma=0$, and by the equivalence of the Gauss and Lebesgue measures on $B_{j}$,

$$
\left\|a_{j}\right\|_{\infty} \leq C \gamma\left(B_{j}\right)^{-1}
$$


Thus the $a_{j}$ are multiples of Gaussian $(1, \infty)$-atoms. Since $a=\sum_{j} \lambda_{j} a_{j}$, we conclude that $a \in H^{1, \infty}(\gamma)$ and

$$
\|a\|_{H^{1, \infty}(\gamma)} \leq C \sum_{j}\left|\lambda_{j}\right| \leq C
$$

\section{The Characterization of $H^{1}(\gamma)$ IN $\mathbb{R}$}

In this section, we shall prove that $f \in H^{1}(\gamma)$ implies $\mathcal{M}_{\text {loc }} f \in L^{1}(\gamma)$ and that, in dimension one, functions in $H^{1}(\gamma)$ can be characterized by the two conditions $\mathcal{M}_{\text {loc }} f \in L^{1}(\gamma)$ and $E(f)<\infty$. We start with a simple but useful lemma dealing with the support of the local grand maximal function.

Lemma 3.1. If $f \in L^{1}(\gamma)$ is supported in the admissible ball $B$, then $\operatorname{supp} \mathcal{M}_{\mathrm{loc}} f$ is contained in the ball $B^{\prime}=B\left(c_{B}, R\right)$, where $R=4 \min \left(1,1 /\left|c_{B}\right|\right)$.

Proof. Let $x \in \operatorname{supp} \mathcal{M}_{\text {loc }} f$. We write $\rho=|x|$ and $c=\left|c_{B}\right|$, so that $B \subset$ $B\left(c_{B}, \min (1,1 / c)\right)$. The balls $B$ and $B(x, \min (1,1 / \rho))$ must intersect, and so

$$
\left|x-c_{B}\right| \leq \min (1,1 / c)+\min (1,1 / \rho) .
$$

To prove the lemma, it is enough to show that

$$
\min (1,1 / \rho) \leq 3 \min (1,1 / c),
$$

since it then follows that $x \in B^{\prime}$. Now $c-\rho \leq\left|x-c_{B}\right|$, so that (3.1) implies

$$
c-\min (1,1 / c) \leq \rho+\min (1,1 / \rho) .
$$

Considering the cases $c \leq 1$ and $c>1$, we conclude from this that

$$
\max (1, c)-\min (1,1 / c) \leq \max (1, \rho)+\min (1,1 / \rho) .
$$

The function $t \mapsto t^{-1}-t, \quad t>0$, and its inverse are clearly decreasing. Considering the values of this function at $t=\min (1,1 / c)$ and $\min (1,1 / \rho) / 3$, we see that (3.2) is equivalent to

$$
\max (1, c)-\min (1,1 / c) \leq 3 \max (1, \rho)-\frac{1}{3} \min (1,1 / \rho) .
$$

Because of (3.3), this inequality follows if

$$
\max (1, \rho)+\min (1,1 / \rho) \leq 3 \max (1, \rho)-\frac{1}{3} \min (1,1 / \rho)
$$

or equivalently $\frac{4}{3} \min (1,1 / \rho) \leq 2 \max (1, \rho)$, which is trivially true. We have proved (3.2) and the lemma.

Lemma 3.2. If $f$ is in $H^{1}(\gamma)$, then $\mathcal{M}_{\mathrm{loc}} f \in L^{1}(\gamma)$ and

$$
\left\|\mathcal{M}_{\text {loc }} f\right\|_{1} \leq C\|f\|_{H^{1}(\gamma)} .
$$

Proof. We shall prove that for any Gaussian atom $a$,

$$
\left\|\mathcal{M}_{\text {loc }} a\right\|_{1} \leq C
$$

from which the lemma follows.

Since (3.4) is obvious if $a$ is the constant function 1, we assume that $a$ is associated to an admissible ball $B$. By the preceding lemma, supp $\mathcal{M}_{\text {loc }} f$ is contained in the ball denoted $B^{\prime}$.

The integral of $\mathcal{M}_{\text {loc }} a$ over $2 B$ with respect to $\gamma$ is no larger than $C$, since $\mathcal{M}_{\text {loc }} a \leq C \sup |a| \leq C / \gamma(B)$. To estimate $\mathcal{M}_{\text {loc }} a$ at a point $x$ in the remaining 
set $B^{\prime} \backslash 2 B$, we take $\phi \in \Phi$ and $0<t<\min (1,1 /|x|)$ and estimate $a * \phi_{t}(x)$. We can assume that $t>d(x, B)$ so that $t>\left|x-c_{B}\right| / 2$, since otherwise $\phi_{t} * a(x)$ will vanish. Write

$$
\phi_{t} * a(x)=t^{-n} \int\left(\phi\left(\frac{x-y}{t}\right)-\phi\left(\frac{x-c_{B}}{t}\right)\right) a(y) \mathrm{d} y+t^{-n} \phi\left(\frac{x-c_{B}}{t}\right) \int a(y) \mathrm{d} y .
$$

Here the first term to the right can be estimated in a standard way by

$$
C t^{-n-1} \int_{B}\left|y-c_{B}\right||a(y)| \mathrm{d} y \leq C\left|x-c_{B}\right|^{-n-1} r_{B} \gamma_{0}\left(c_{B}\right)^{-1} .
$$

To deal with the second term, we estimate $\int a(y) \mathrm{d} y$, knowing that the integral of $a$ against $\gamma$ vanishes. Thus

$$
\int a(y) \mathrm{d} y=\int a(y) \frac{\gamma_{0}\left(c_{B}\right)-\gamma_{0}(y)}{\gamma_{0}\left(c_{B}\right)} \mathrm{d} y
$$

The fraction appearing here is

$$
e^{\left|c_{B}\right|^{2}}\left(e^{-\left|c_{B}\right|^{2}}-e^{-|y|^{2}}\right)=1-e^{\left(c_{B}-y\right) \cdot\left(c_{B}+y\right)},
$$

and the last exponent stays bounded for $y \in B$. Thus the modulus of the right-hand side of (3.6) is at most $C\left|c_{B}-y\right|\left|c_{B}+y\right| \leq C r_{B}\left(1+\left|c_{B}\right|\right)$. Since $\int|a| d \gamma \leq 1$, this implies that

$$
\left|\int a(y) \mathrm{d} y\right| \leq C r_{B}\left(1+\left|c_{B}\right|\right) \gamma_{0}\left(c_{B}\right)^{-1}
$$

For the last term in (3.5), we thus get the bound $C\left|x-c_{B}\right|^{-n} r_{B}\left(1+\left|c_{B}\right|\right) \gamma_{0}\left(c_{B}\right)^{-1}$.

Putting things together, we conclude that for $x \in B^{\prime} \backslash 2 B$,

$$
\mathcal{M}_{\text {loc }} a(x) \leq C\left|x-c_{B}\right|^{-n-1} r_{B} \gamma_{0}\left(c_{B}\right)^{-1}+C\left|x-c_{B}\right|^{-n} r_{B}\left(1+\left|c_{B}\right|\right) \gamma_{0}\left(c_{B}\right)^{-1} \text {. }
$$

An integration with respect to $d \gamma$, or equivalently $\gamma_{0} d \lambda$, then leads to

$$
\int_{B^{\prime} \backslash 2 B} \mathcal{M}_{\text {loc }} a(x) \mathrm{d} \gamma(x) \leq C+C r_{B}\left(1+\left|c_{B}\right|\right) \log \frac{\min \left(1,1 /\left|c_{B}\right|\right)}{r_{B}} \leq C,
$$

and (3.4) is proved.

Theorem 3.3. Let $n=1$, and suppose that $f$ is a function in $L^{1}(\gamma)$. Then $f$ is in $H^{1}(\gamma)$ if and only if $\mathcal{M}_{\mathrm{loc}} f \in L^{1}(\gamma)$ and $E(f)<\infty$. The norms $\|f\|_{H^{1}(\gamma)}$ and $\left\|\mathcal{M}_{\text {loc }} f\right\|_{L^{1}(\gamma)}+E(f)$ are equivalent.

Proof. Suppose that $f \in H^{1}(\gamma)$. Then $\mathcal{M}_{\text {loc }} f \in L^{1}(\gamma)$ by Lemma 3.2. To prove the necessity of the condition $E(f)<\infty$, it suffices to show that $E(a)<C$ for all Gaussian atoms $a$. This is obvious for the exceptional atom 1. If $a$ is associated to a ball $B \in \mathcal{B}_{1}$, it follows from the inequality

$$
\left|\int_{-\infty}^{-x} a \mathrm{~d} \gamma\right|+\left|\int_{x}^{\infty} a \mathrm{~d} \gamma\right| \leq \mathbf{1}_{(-B) \cup B}(x) .
$$

Conversely, assume that $f$ is a function in $L^{1}(\gamma)$ such that $\mathcal{M}_{\text {loc }} f \in L^{1}(\gamma)$ and $E(f)<\infty$. We shall prove that $f \in H^{1}(\gamma)$, by constructing a Gaussian atomic decomposition $f=\sum_{j} \lambda_{j} a_{j}$ such that $\sum_{j}\left|\lambda_{j}\right| \leq C\left(\left\|\mathcal{M}_{\text {loc }} f\right\|_{1}+E(f)\right)$.

Most of the following argument, up to the decomposition (3.15), works also in the $n$-dimensional setting. Since we shall need it in the next section, we carry out that part in $\mathbb{R}^{n}$. 
By subtracting a multiple of the exceptional atom 1, we may without loss of generality assume that

$$
\int f \mathrm{~d} \gamma=0
$$

Let $\left\{B_{j}\right\}$ be a covering of $\mathbb{R}^{n}$ by maximal admissible balls. We can choose this covering in such way that the family $\left\{\frac{1}{2} B_{j}\right\}$ is disjoint and $\left\{4 B_{j}\right\}$ has bounded overlap [10, Lemma 2.4]. Fix a smooth nonnegative partition of unity $\left\{\eta_{j}\right\}$ in $\mathbb{R}^{n}$ such that $\operatorname{supp} \eta_{j} \subset B_{j}$ and $\eta_{j}=1$ on $\frac{1}{2} B_{j}$ and verifying $\left|\nabla \eta_{j}\right| \leq C / r_{B_{j}}$. Thus $f=\sum_{j} f \eta_{j}$. We now need the following lemma.

Lemma 3.4. For $g$ in $L_{\mathrm{loc}}^{1}(\gamma)$ and $x \in \mathbb{R}^{n}$ one has

$$
\mathcal{M}_{\text {loc }}\left(g \eta_{j} \gamma_{0}\right)(x) \leq C \gamma_{0}\left(c_{B_{j}}\right) \mathcal{M}_{\text {loc }} g(x) \mathbf{1}_{4 B_{j}}(x) \quad \forall j .
$$

Proof. Since the support of $\eta_{j}$ is contained in $B_{j}$, the support of $\mathcal{M}_{\mathrm{loc}}\left(g \eta_{j} \gamma_{0}\right)$ is contained in the ball $4 B_{j}$, because of Lemma 3.1. Moreover, for $\phi \in \Phi$ and $x \in 4 B_{j}$,

$$
\phi_{t} *\left(g \eta_{j} \gamma_{0}\right)(x)=\gamma_{0}\left(c_{B_{j}}\right) \tilde{\phi}_{t} * g(x),
$$

where $\tilde{\phi}(z)=\phi(z) \eta_{j}(x-t z) \gamma_{0}(x-t z) / \gamma_{0}\left(c_{B_{j}}\right)$. Thus, to prove (3.8) it suffices to show that there exists a positive constant $C$ such that $\tilde{\phi} \in C \Phi$ for $x \in 4 B_{j}$ and $0<t<\min (1,1 /|x|)$. The support of $\tilde{\phi}$ is contained in $B(0,1)$ and

$$
|\tilde{\phi}(z)| \leq \frac{\gamma_{0}(x-t z)}{\gamma_{0}\left(c_{B_{j}}\right)} \leq C
$$

because for $|z| \leq 1$,

$$
\left|x-t z-c_{B_{j}}\right| \leq\left|x-c_{B_{j}}\right|+|t z| \leq C \min \left(1,1 /\left|c_{B_{j}}\right|\right) .
$$

Similarly $|\nabla \tilde{\phi}(z)| \leq C$, because the gradients $\nabla_{z} \eta_{j}(x-t z)$ and $\nabla_{z} \gamma_{0}(x-t z) / \gamma_{0}\left(c_{B_{j}}\right)$ give the factors $t\left(1+\left|c_{B_{j}}\right|\right)$ and $t|x-t z| \gamma_{0}(x-t z) / \gamma_{0}\left(c_{B_{j}}\right)$, respectively, both of which are bounded. This concludes the proof of Lemma 3.4

Continuing the proof of Theorem 3.3, we define $b_{j} \in \mathbb{C}$ for each $j \in \mathbb{N}$ by

$$
\int_{-\infty}^{\infty}\left(f-b_{j}\right) \eta_{j} \mathrm{~d} \gamma=0
$$

Note that since $\eta_{j}=1$ on $\frac{1}{2} B_{j}$,

$$
\left|b_{j}\right|=\left|\frac{\int f \eta_{j} \mathrm{~d} \gamma}{\int \eta_{j} \mathrm{~d} \gamma}\right| \leq C \frac{1}{\gamma\left(B_{j}\right)} \int_{B_{j}}|f| \mathrm{d} \gamma .
$$

We now apply Lemma 3.4 with $g=f-b_{j}$ and use the subadditivity of $\mathcal{M}_{\text {loc }}$ combined with (3.10) to get

$$
\begin{aligned}
\int \mathcal{M}_{\mathrm{loc}}\left(\left(f-b_{j}\right) \eta_{j} \gamma_{0}\right) \mathrm{d} \lambda & \leq C \int_{4 B_{j}} \mathcal{M}_{\mathrm{loc}} f \gamma_{0}\left(c_{B_{j}}\right) \mathrm{d} \lambda+C \frac{\gamma\left(4 B_{j}\right)}{\gamma\left(B_{j}\right)} \int_{B_{j}}|f| \mathrm{d} \gamma \\
& \leq C \int_{4 B_{j}} \mathcal{M}_{\mathrm{loc}} f \mathrm{~d} \gamma
\end{aligned}
$$

Lemma 3.5. The function $\left(f-b_{j}\right) \eta_{j} \gamma_{0}$ is in $H^{1}(\lambda)$ and

$$
\left\|\left(f-b_{j}\right) \eta_{j} \gamma_{0}\right\|_{H^{1}(\lambda)} \leq \int_{4 B_{j}} \mathcal{M}_{\mathrm{loc}} f \mathrm{~d} \gamma .
$$


Proof. By the maximal characterization of the classical space $H^{1}(\lambda)$, it suffices to show that

$$
\int \mathcal{M}\left(\left(f-b_{j}\right) \eta_{j} \gamma_{0}\right) \mathrm{d} \lambda(x) \leq C \int_{4 B_{j}} \mathcal{M}_{\mathrm{loc}} f \mathrm{~d} \gamma .
$$

Because of (3.11), all that needs to be verified is that

$$
\int \sup _{\phi \in \Phi} \sup _{t \geq \min (1,1 /|x|)}\left|\left(\left(f-b_{j}\right) \eta_{j} \gamma_{0}\right) * \phi_{t}(x)\right| \mathrm{d} \lambda(x) \leq C \int_{4 B_{j}} \mathcal{M}_{\text {loc }} f \mathrm{~d} \gamma .
$$

To prove (3.14), we split the integral in the left-hand side into the sum

$$
\int_{4 B_{j}} \cdots \mathrm{d} \lambda(x)+\int_{\left(4 B_{j}\right)^{c}} \cdots \mathrm{d} \lambda(x) .
$$

If $x \in 4 B_{j}$, then for $\phi \in \Phi$ and $t \geq \min (1,1 /|x|)$,

$$
\begin{aligned}
\left|\phi_{t} *\left(\left(f-b_{j}\right) \eta_{j} \gamma_{0}\right)(x)\right| & \leq t^{-n} \int_{B_{j}}\left|f(y)-b_{j}\right| \mathrm{d} \gamma(y) \\
& \leq(1+|x|)^{n} \int_{B_{j}}\left(|f(y)|+\left|b_{j}\right|\right) \mathrm{d} \gamma(y) \\
& \leq C\left(1+\left|c_{B_{j}}\right|\right)^{n} \int_{B_{j}}|f(y)| \mathrm{d} \gamma(y),
\end{aligned}
$$

the last inequality because of (3.10). Hence

$$
\int_{4 B_{j}} \cdots \mathrm{d} \lambda(x) \leq C\left|4 B_{j}\right|\left(1+\left|c_{B_{j}}\right|\right)^{n} \int_{B_{j}}|f| \mathrm{d} \gamma \leq C \int_{B_{j}} \mathcal{M}_{\mathrm{loc}} f \mathrm{~d} \gamma .
$$

If $x \in\left(4 B_{j}\right)^{c}$, we take $\phi$ and $t$ as before and observe that we can assume that $t>d\left(x, B_{j}\right)$, since otherwise the convolution in (3.14) will vanish. In view of (3.9) and (3.10), we then get

$$
\begin{aligned}
\left|\phi_{t} *\left(\left(f-b_{j}\right) \eta_{j} \gamma_{0}\right)(x)\right| & \leq \int_{B_{j}}\left|\phi_{t}(x-y)-\phi_{t}\left(x-c_{B_{j}}\right)\right|\left|f(y)-b_{j}\right| \eta_{j}(y) \mathrm{d} \gamma(y) \\
& \leq C t^{-n-1} \int_{B_{j}}\left|y-c_{B_{j}}\right|\left|f(y)-b_{j}\right| \mathrm{d} \gamma(y) \\
& \leq C \frac{1}{d\left(x, B_{j}\right)^{n+1}} r_{B_{j}} \int_{B_{j}}|f(y)| \mathrm{d} \gamma(y) .
\end{aligned}
$$

This implies that

$$
\int_{\left(4 B_{j}\right)^{c}} \cdots \mathrm{d} \lambda(x) \leq C \int_{B_{j}}|f| \mathrm{d} \gamma \leq C \int_{B_{j}} \mathcal{M}_{\mathrm{loc}} f \mathrm{~d} \gamma
$$

We have proved (3.14) and the lemma.

We can now finish the proof of Theorem 3.3. By Lemmata 3.5 and 2.1, each function $\left(f-b_{j}\right) \eta_{j} \gamma_{0}$ has an atomic decomposition $\sum_{k} \lambda_{j k} \alpha_{j k}$, where the $\alpha_{j k}$ are Lebesgue atoms with supports in $2 B_{j}$ and

$$
\sum_{k}\left|\lambda_{j k}\right| \leq C \int_{4 B_{j}} \mathcal{M}_{\mathrm{loc}} f \mathrm{~d} \gamma
$$


As we saw in the proof of Theorem 2.2, each $a_{j k}=\gamma_{0}^{-1} \alpha_{j k}$ is a multiple of a Gaussian atom, with a factor which is independent of $j$ and $k$. Thus

$$
f=\sum_{j}\left(f-b_{j}\right) \eta_{j}+\sum_{j} b_{j} \eta_{j}=\sum_{j} \sum_{k} \lambda_{j k} a_{j k}+\sum_{j} b_{j} \eta_{j}
$$

and

$$
\sum_{j, k}\left|\lambda_{j k}\right| \leq \sum_{j} \int_{4 B_{j}} \mathcal{M}_{\mathrm{loc}} f \mathrm{~d} \gamma \leq C\left\|\mathcal{M}_{\mathrm{loc}} f\right\|_{L^{1}(\gamma)}
$$

To complete the proof of Theorem 3.3 , we need to find an atomic decomposition of $\sum_{j} b_{j} \eta_{j}$. It is here that we must restrict ourselves to the one-dimensional case and that the global condition $E(f)<\infty$ plays a role.

Choose the intervals $I_{0}=(-1,1), I_{j}=(\sqrt{j-1}, \sqrt{j+1})$ for $j \geq 1$ and $I_{j}=-I_{|j|}$ for $j \leq-1$. The intervals $I_{j}$ have essentially the same properties as the balls $B_{j}$ introduced above, and we can use them instead of the $B_{j}$ to construct $\eta_{j}$ and $b_{j}$ as before. To decompose now $\sum_{j} b_{j} \eta_{j}$, we first normalise the functions $\eta_{j}$, letting

$$
\tilde{\eta}_{j}=\frac{\eta_{j}}{\int \eta_{j} \mathrm{~d} \gamma}
$$

Then $b_{j} \eta_{j}=\int f \eta_{j} \mathrm{~d} \gamma \tilde{\eta}_{j}$, and clearly

$$
\sum_{j \geq k} \int f \eta_{j} \mathrm{~d} \gamma=\int f \mu_{k} \mathrm{~d} \gamma, \quad k \in \mathbb{Z}
$$

where $\mu_{k}(x)=\sum_{j \geq k} \eta_{j}(x)$. Notice that $\int f \mu_{k} \mathrm{~d} \gamma \rightarrow 0$ as $k \rightarrow \pm \infty$, in view of (3.7). A summation by parts now yields

$$
\sum_{j \in \mathbb{Z}} \int f \eta_{j} \mathrm{~d} \gamma \tilde{\eta}_{j}=\sum_{k \in \mathbb{Z}} \int f \mu_{k} \mathrm{~d} \gamma\left(\tilde{\eta}_{k}-\tilde{\eta}_{k-1}\right)
$$

But $\tilde{\eta}_{k}-\tilde{\eta}_{k-1}$ is $C$ times a Gaussian atom if we use admissible balls at some scale $s>1$ in the definition of atoms. Thus (3.16) is our desired atomic decomposition of $\sum_{j} b_{j} \eta_{j}$, provided we can estimate the coefficients by showing that

$$
\sum_{k \in \mathbb{Z}}\left|\int f \mu_{k} \mathrm{~d} \gamma\right| \leq C\left(\|f\|_{1}+E(f)\right)
$$

To this end, observe that

$$
\int f \mu_{k} \mathrm{~d} \gamma=\int f(x) \int_{-\infty}^{x} \mu_{k}^{\prime}(y) \mathrm{d} \lambda(y) \mathrm{d} \gamma(x)=\int \mu_{k}^{\prime}(y) \int_{y}^{\infty} f(x) \mathrm{d} \gamma(x) \mathrm{d} \lambda(y) .
$$

Since the support of $\mu_{k}^{\prime}$ is contained in $I_{k}$ and

$$
\left|\mu_{k}^{\prime}(y)\right| \leq \frac{C}{\left|I_{k}\right|} \leq C\left(1+\left|c_{I_{k}}\right|\right)
$$


we obtain, using also the bounded overlap of the $I_{j}$,

$$
\begin{aligned}
\sum_{k \in \mathbb{Z}}\left|\int f \mu_{k} \mathrm{~d} \gamma\right| & \leq C \sum_{k} \int_{I_{k}}\left(1+\left|c_{I_{k}}\right|\right)\left|\int_{y}^{\infty} f(x) \mathrm{d} \gamma(x)\right| \mathrm{d} \lambda(y) \\
& \leq C \int_{-\infty}^{\infty}(1+|y|)\left|\int_{y}^{\infty} f \mathrm{~d} \gamma\right| \mathrm{d} \lambda(y) \\
& =C \int_{0}^{\infty}(1+y)\left(\left|\int_{y}^{\infty} f \mathrm{~d} \gamma\right|+\left|\int_{-y}^{\infty} f \mathrm{~d} \gamma\right|\right) \mathrm{d} \lambda(y) \\
& \leq C\left(\|f\|_{1}+E(f)\right) ;
\end{aligned}
$$

here we used (3.7). This concludes the proof of Theorem 3.3

\section{A Characterization of NOnNEgative Functions in $H^{1}(\gamma)$}

The dimension $n$ is now arbitrary. The following lemma will be needed.

Lemma 4.1. Let $\phi_{0}=\gamma(B(0,1))^{-1} \mathbf{1}_{B(0,1)}$. If $g \in L^{\infty}$ is supported in a maximal admissible ball $B$, then

$$
\left\|g-\int g \mathrm{~d} \gamma \phi_{0}\right\|_{H^{1}(\gamma)} \leq C\left(1+\left|c_{B}\right|^{2}\right) \gamma(B)\|g\|_{L^{\infty}} .
$$

Proof. We shall construct atoms whose supports form a chain connecting $B(0,1)$ to $B$. First we define a finite sequence of maximal admissible balls

$$
\tilde{B}_{0}=B(0,1), \tilde{B}_{1}, \ldots, \tilde{B}_{N},
$$

all with centres $c_{\tilde{B}_{j}}$ on the segment $\left[0, c_{B}\right]$. The absolute values $\rho_{j}=\left|c_{\tilde{B}_{j}}\right|$ shall be increasing in $j$, and the boundary $\partial \tilde{B}_{j}$ shall contain $c_{\tilde{B}_{j-1}}$ for $j=1, \ldots, N-1$, which means that

$$
\rho_{j}-\frac{1}{\rho_{j}}=\rho_{j-1}, \quad j=1, \ldots, N-1,
$$

and $\rho_{0}=0, \quad \rho_{1}=1$. Finally, $N$ is defined so that $\tilde{B}_{N-1}$ is the first ball of the sequence that contains $c_{B}$, and $\tilde{B}_{N}=B$. Squaring (4.1), we get

$$
\rho_{j}^{2}-\rho_{j-1}^{2}=2-\frac{1}{\rho_{j}^{2}} \geq 1
$$

so that $\rho_{N-1}^{2} \geq N-1$. It follows that

$$
N \leq\left|c_{B}\right|^{2}+1
$$

Next, we denote by $B_{j}, j=1, \ldots, N$, the largest ball contained in $\tilde{B}_{j} \cap \tilde{B}_{j-1}$. Notice that the three balls $\tilde{B}_{j}, \tilde{B}_{j-1}$ and $B_{j}$ have comparable radii and comparable Gaussian measures. Now define functions $\phi_{j}$ and $g_{j}$ by setting

$$
\begin{aligned}
\phi_{j} & =\gamma\left(B_{j}\right)^{-1} \mathbf{1}_{B_{j}} \quad j=1, \ldots, N, \\
g_{j} & =\int g \mathrm{~d} \gamma\left(\phi_{j}-\phi_{j-1}\right), \quad j=1, \ldots, N, \\
g_{N+1} & =g-\int g \mathrm{~d} \gamma \phi_{N} .
\end{aligned}
$$


Clearly,

$$
g-\int g \mathrm{~d} \gamma \phi_{0}=\sum_{j=1}^{N+1} g_{j}
$$

Each function $g_{j}$ is a multiple of an atom. Indeed, its integral against $\gamma$ vanishes. Moreover, if $1 \leq j \leq N$, the support of $g_{j}$ is contained in $\tilde{B}_{j-1}$ and

$$
\left\|g_{j}\right\|_{\infty} \leq\left(\gamma\left(B_{j}\right)^{-1}+\gamma\left(B_{j-1}\right)^{-1}\right) \int|g| \mathrm{d} \gamma \leq C \gamma\left(\tilde{B}_{j-1}\right)^{-1} \gamma(B)\|g\|_{L^{\infty}} .
$$

The support of $\phi_{N+1}$ is contained in $B$ and

$$
\left\|g_{N+1}\right\|_{\infty} \leq\|g\|_{\infty}+\gamma(B)^{-1} \int|g| \mathrm{d} \gamma \leq C\|g\|_{L^{\infty}} .
$$

Thus

$$
\left\|g_{j}\right\|_{H^{1}(\gamma)} \leq C \gamma(B)\|g\|_{L^{\infty}}, \quad j=1, \ldots, N+1 .
$$

Summing the coefficients in the atomic decomposition (4.3), we then obtain via (4.2),

$$
\left\|g-\int g \mathrm{~d} \gamma \phi_{0}\right\|_{H^{1}(\gamma)} \leq C(N+1) \gamma(B)\|g\|_{L^{\infty}} \leq C\left(1+\left|c_{B}\right|^{2}\right) \gamma(B)\|g\|_{L^{\infty}} .
$$

The proof of the lemma is complete.

Theorem 4.2. Suppose that $f$ is a function in $L^{1}(\gamma)$. If $\mathcal{M}_{\mathrm{loc}} f$ is in $L^{1}(\gamma)$ and

$$
E_{+}(f)=\int|x|^{2}|f(x)| \mathrm{d} \gamma(x)<\infty,
$$

then $f$ is in $H^{1}(\gamma)$ and

$$
\|f\|_{H^{1}(\gamma)} \leq C\left\|\mathcal{M}_{\text {loc }} f\right\|_{1}+C E_{+}(f) .
$$

If $f$ is nonnegative, the conditions $\mathcal{M}_{\mathrm{loc}} f \in L^{1}(\gamma)$ and $E_{+}(f)<\infty$ are also necessary for $f$ to be in $H^{1}(\gamma)$.

Proof. Let $f$ be a function in $L^{1}(\gamma)$ such that $\mathcal{M}_{\text {loc }} f \in L^{1}(\gamma)$ and $E_{+}(f)<\infty$. Write $f=c(f)+f_{0}$, where $c(f)=\int f \mathrm{~d} \gamma$. Since $c(f)$ is a multiple of the exceptional atom, it suffices to find an atomic decomposition of $f_{0}$. Note that $f_{0}$ satisfies

$$
\mathcal{M}_{\text {loc }} f_{0} \in L^{1}(\gamma) \quad \text { and } \quad \int|x|^{2}\left|f_{0}(x)\right| \mathrm{d} \gamma(x)<\infty .
$$

Let $\left\{B_{j}\right\}$ be the covering of $\mathbb{R}^{n}$ by maximal admissible balls and $\left\{\eta_{j}\right\}$ the corresponding partition of unity introduced in the proof of Theorem 3.3. As there, we choose numbers $b_{j} \in \mathbb{C}$ such that

$$
\int_{-\infty}^{\infty}\left(f_{0}-b_{j}\right) \eta_{j} \mathrm{~d} \gamma=0 \quad \forall j
$$

Then the argument leading to (3.15) shows that

$$
f_{0}=\sum_{j} \sum_{k} \lambda_{j k} a_{j k}+\sum_{j} b_{j} \eta_{j}
$$

where the $a_{j k}$ are Gaussian atoms supported in $4 B_{j}$ and

$$
\sum_{j, k}\left|\lambda_{j k}\right| \leq C\left\|\mathcal{M}_{\text {loc }} f_{0}\right\|_{L^{1}(\gamma)}
$$


It remains only to prove that $\sum_{j} b_{j} \eta_{j}$ is in $H^{1}(\gamma)$. We write $g_{j}=b_{j} \eta_{j}$ and observe that

$$
\int \sum_{j} g_{j} \mathrm{~d} \gamma=0
$$

because $f_{0}$ and the $a_{i j}$ have integrals zero. Thus

$$
\sum_{j} g_{j}=\sum_{j}\left(g_{j}-\int g_{j} \mathrm{~d} \gamma \phi_{0}\right),
$$

where $\phi_{0}$ is as in Lemma 4.1. Since (3.10) remains valid for $f_{0}$, we have

$$
\left\|g_{j}\right\|_{\infty} \leq C \frac{1}{\gamma\left(B_{j}\right)} \int_{B_{j}}\left|f_{0}\right| \mathrm{d} \gamma
$$

Lemma 4.1 thus applies to each $g_{j}$, and using also the bounded overlap of the $B_{j}$ we conclude

$$
\left\|\sum_{j} g_{j}\right\|_{H^{1}(\gamma)} \leq C \sum_{j}\left(1+\left|c_{B_{j}}\right|^{2}\right) \int_{B_{j}}\left|f_{0}\right| \mathrm{d} \gamma \leq C \int\left(1+|x|^{2}\right)\left|f_{0}\right| \mathrm{d} \gamma .
$$

This concludes the proof of the sufficiency and the norm estimate.

The necessity of the condition $\mathcal{M}_{\text {loc }} f \in L^{1}(\gamma)$ was obtained in Lemma 3.2

To prove the necessity of (4.4), let $0 \leq f \in H^{1}(\gamma)$. We first observe that the function $x \mapsto|x|^{2}$ is in $B M O(\gamma)$. Indeed, its oscillation on any admissible ball is bounded. Since $B M O(\gamma)$ is a lattice, the functions $g_{k}(x)=\min \left\{|x|^{2}, k\right\}$ are in $B M O(\gamma)$, uniformly for $k \geq 1$. By the monotone convergence theorem and the duality between $H^{1}(\gamma)$ and $B M O(\gamma)$,

$$
\int|x|^{2} f(x) \mathrm{d} \gamma(x)=\lim _{k} \int g_{k}(x) f(x) \mathrm{d} \gamma(x) \leq C\|f\|_{H^{1}(\gamma)} .
$$

The theorem is proved.

The following result is a noteworthy consequence of Theorem 4.2

Corollary 4.3. For $1<p \leq \infty$, one has continuous inclusions $L^{p}(\gamma) \subset H^{1}(\gamma)$ and $B M O(\gamma) \subset L^{p^{\prime}}(\gamma)$, where $p^{\prime}=p /(p-1)$.

Proof. We claim that the operator $\mathcal{M}_{\text {loc }}$ is bounded on $L^{p}(\gamma)$ for $1<p \leq \infty$. Deferring momentarily the proof of this claim, we complete the proof of the corollary. Suppose that $f$ is in $L^{p}(\gamma)$. Then $\mathcal{M}_{\text {loc }} f$ is in $L^{1}(\gamma)$, because

$$
\left\|\mathcal{M}_{\text {loc }} f\right\|_{1} \leq\left\|\mathcal{M}_{\text {loc }} f\right\|_{p} \leq C\|f\|_{p}<\infty,
$$

since $\gamma\left(\mathbb{R}^{n}\right)=1$. Moreover, $E_{+}(f) \leq\left\||x|^{2}\right\|_{p^{\prime}}\|f\|_{p}<\infty$, by Hölder's inequality. Thus $f \in H^{1}(\gamma)$ by Theorem 4.2. It also follows that the inclusion $L^{p}(\gamma) \subset H^{1}(\gamma)$ is continuous, and by duality we get the continuous inclusion $B M O(\gamma) \subset L^{p^{\prime}}(\gamma)$.

It remains to prove the claim. We shall use again the covering $\left\{B_{j}\right\}$ from the proof of Theorem 3.3. First we observe that the inequality

$$
\left\|\mathcal{M}_{\text {loc }} g\right\|_{p} \leq C\|g\|_{p}
$$

holds when supp $g \subset B_{j}$, with a constant $C$ independent of $j$. Indeed, $\mathcal{M}_{\text {loc }}$ is bounded on $L^{p}(\lambda)$, and $\mathcal{M}_{\text {loc }} g$ is supported in the ball $4 B_{j}$, where the Gaussian measure is essentially proportional to $d \lambda$. 
Given a function $f \in L^{p}(\gamma)$, we write it as a sum $f=\sum f_{j}$ with $\operatorname{supp} f_{j} \subset B_{j}$ and with the sets $\left\{f_{j} \neq 0\right\}$ pairwise disjoint. We can then apply (4.7) to each $f_{j}$ and sum.

\section{ACKNOWLEDGEMENT}

The authors thank J. Dziubański for a useful discussion about Lemma 2.1.

\section{REFERENCES}

[1] A. Carbonaro, G. Mauceri and S. Meda, $H^{1}$ and $B M O$ on certain measured metric spaces, Ann. Sc. Norm. Super. Pisa Cl. Sci. (5) 8 (2009), no. 3, 543-582. MR2581426(2010j:42025)

[2] A. Carbonaro, G. Mauceri and S. Meda, $H^{1}$ and $B M O$ for certain locally doubling metric measure spaces of finite measure, Colloq. Math. 118 (2010), 13-41. MR2600517 (2011b:42034)

[3] A. Carbonaro, G. Mauceri and S. Meda, Comparison of spaces of Hardy type for the OrnsteinUhlenbeck operator, Potential Anal. 33 (2010), 85-105. MR2644215(2011k:42045)

[4] D.-C. Chang, S. G. Krantz and E. M. Stein, $H^{p}$ theory on a smooth domain in $\mathbb{R}^{n}$ and elliptic boundary value problems, J. Funct. Anal. 114 (1993), 286-347. MR.1223705 (94j:46032)

[5] R. Coifman, Y. Meyer and E.M. Stein, Some new function spaces and their applications to harmonic analysis, J. Funct. Anal. 62 (1985), 304-335. MR791851 (86i:46029)

[6] R. R. Coifman and G. Weiss, Extensions of Hardy spaces and their use in analysis, Bull. Amer. Math. Soc. 83 (1977), 569-645. MR0447954 (56:6264)

[7] O. Dragicevic and A. Volberg, Bellman functions and dimensionless estimates of LittlewoodPaley type, J. Oper. Theory 56 (2006), 167-198. MR2261616 (2007g:42023)

[8] E. B. Fabes, C. Gutiérrez and R. Scotto, Weak type estimates for the Riesz transforms associated with the Gaussian measure, Rev. Mat. Iberoamericana 10 (1994), 229-281. MR 1286476 (95m:42023)

[9] L. Forzani and R. Scotto, The higher order Riesz transforms for Gaussian measure need not be weak type (1,1), Studia Math. 131 (1998), 205-214. MR1644460 (99h:42034)

[10] J. García-Cuerva, G. Mauceri, P. Sjögren and J.L. Torrea, Higher order Riesz operators for the Ornstein-Uhlenbeck semigroup, Potential Anal. 10 (1999), 379-407. MR1698617 (2000j:42024)

[11] J. García-Cuerva, G. Mauceri, P. Sjögren and J.L. Torrea, Spectral multipliers for the Ornstein-Uhlenbeck semigroup, J. Anal. Math. 78 (1999), 281-305. MR1714425 (2000m:42015)

[12] J. García-Cuerva, G. Mauceri, S. Meda, P. Sjögren and J. L. Torrea, Functional calculus for the Ornstein-Uhlenbeck operator, J. Funct. Anal. 183 (2001), no. 2, 413-450. MR1844213 (2002d:47023)

[13] J. García-Cuerva, G. Mauceri, S. Meda, P. Sjögren and J.L. Torrea, Maximal operators for the Ornstein-Uhlenbeck semigroup, J. London Math. Soc. 67 (2003), 219-234. MR1942422 (2003k:47048)

[14] R.F. Gundy, Sur les transformations de Riesz pour le semigroupe d'Ornstein-Uhlenbeck, $C . R$. Acad. Sci. Paris Sci. Ser. I Math. 303 (1986), 967-970. MR.877182 (88c:60108)

[15] C. E. Gutiérrez, C. Segovia and J. L. Torrea, On higher order Riesz transforms for Gaussian measures, J. Fourier Anal. Appl. 2 (1996), 583-596. MR1423529 (98m:42017)

[16] C. E. Gutiérrez and W. Urbina, Estimates for the maximal operator of the OrnsteinUhlenbeck semigroup, Proc. Amer. Math. Soc. 113 (1991), no. 1, 99-104. MR.1068123 (92a:42023)

[17] C. E. Gutiérrez, On the Riesz transforms for Gaussian measures, J. Funct. Anal. 120 (1994), 107-134. MR 1262249 (95c:35013)

[18] W. Hebisch, G. Mauceri and S. Meda, Holomorphy of spectral multipliers of the OrnsteinUhlenbeck operator, J. Funct. Anal. 210 (2004), 101-124. MR2052115 (2005g:47024)

[19] L. Liu and D. Yang, Characterizations of BMO associated with Gauss measures via commutators of local fractional integrals, Israel J. Math. 180 (2010), 285-315. MR2735067 (2011k:42049)

[20] L. Liu and D. Yang, BLO spaces associated with the Ornstein-Uhlenbeck operator, Bull. Sci. Math. 132 (2008), 633-649. MR2474485(2010c:42045) 
[21] J. Maas, J. van Neerven and P. Portal, Conical square functions and non-tangential maximal functions with respect to the Gaussian measure, Publ. Mat. 55 (2011), no. 2, 313-341. MR2839445

[22] J. Maas, J. van Neerven and P. Portal, Whitney coverings and the tent spaces $T^{1, q}(\gamma)$ for the Gaussian measure, arXiv: 1002.4911v1 [math.FA], to appear in Ark. Mat.

[23] R. A. Macías and C. Segovia, A decomposition into atoms of distributions on spaces of homogeneous type, Adv. in Math. 33 (1979), 271-309. MR546296 (81c:32017b)

[24] G. Mauceri and S. Meda, BMO and $H^{1}$ for the Ornstein-Uhlenbeck operator, J. Funct. Anal. 252 (2007), 278-313. MR2357358 (2008m:42024)

[25] G. Mauceri, S. Meda and P. Sjögren, Sharp estimates for the Ornstein-Uhlenbeck operator, Ann. Sc. Norm. Sup. Pisa, Classe di Scienze, Serie V, 3 (2004), n. 3, 447-480. MR2099246 (2005j:47042)

[26] G. Mauceri, S. Meda and P. Sjögren, Endpoint estimates for first-order Riesz transforms associated to the Ornstein-Uhlenbeck operator, Rev. Mat. Iberoam. 28 (2012), no. 1, 77-91. MR.2904131

[27] T. Menárguez, S. Pérez and F. Soria, The Mehler maximal function: a geometric proof of the weak type 1, J. London Math. Soc. (2) 61 (2000), 846-856. MR1766109 (2001i:42029)

[28] P. A. Meyer, Transformations de Riesz pour le lois Gaussiennes, Springer Lecture Notes in Mathematics 1059 (1984), 179-193. MR770960 (86i:60150)

[29] B. Muckenhoupt, Hermite conjugate expansions, Trans. Amer. Math. Soc. 139 (1969), 243260. MR0249918 (40:3159)

[30] S. Pérez, The local part and the strong type for operators related to the Gauss measure, J. Geom. Anal. 11 (2001), no. 3, 491-507. MR.1857854 (2002h:42027)

[31] S. Pérez and F. Soria, Operators associated with the Ornstein-Uhlenbeck semigroup, J. London Math. Soc. 61 (2000), 857-871. MR.1766110 (2001i:42030)

[32] G. Pisier, Riesz transforms: a simpler analytic proof of P. A. Meyer's inequality, Springer Lecture Notes in Mathematics 1321 (1988), 485-501. MR960544 (89m:60178)

[33] P. Sjögren, On the maximal function for the Mehler kernel, in Harmonic Analysis, Cortona, 1982, Springer Lecture Notes in Mathematics 992 (1983), 73-82. MR729346 (85j:35031)

[34] E.M. Stein, Harmonic Analysis. Real variable methods, orthogonality and oscillatory integrals, Princeton Math. Series No. 43, Princeton, N.J., 1993. MR.1232192 (95c:42002)

[35] W. Urbina, On singular integrals with respect to the Gaussian measure, Ann. Sc. Norm. Sup. Pisa, Classe di Scienze, Serie IV, 17 (1990), no. 4, 531-567. MR.1093708 (92d:42010)

Dipartimento di Matematica, Università di Genova, via Dodecaneso 35, 16146 Genova, ITALIA

E-mail address: mauceri@dima.unige.it

Dipartimento di Matematica e Applicazioni, Università di Milano-Bicocca, Via

R. Cozzi 53, 20125 Milano, Italy

E-mail address: stefano.meda@unimib.it

Mathematical Sciences, University of Gothenburg, Box 100, S-405 30 Gothenburg, Sweden - And - Mathematical Sciences, Chalmers University of Technology, SE-412 96 Gothenburg, Sweden

E-mail address: peters@chalmers.se 